\title{
A descriptive cross-sectional study: knowledge, attitude and practice on telemedicine for healthcare among the general public in the western province of Sri Lanka
}

\author{
D.H. Jayakody \\ Postgraduate Institute of Medicine, University of Colombo, Sri Lanka \\ harsha_jayakody@yahoo.com \\ https://orcid.org/0000-0001-9151-3968
}

\begin{abstract}
Introduction: In the era of technology, most service fields have digitalized their systems to provide a more convenient, faster and safer service of better quality to their clients. However, Sri Lankans have not adapted to the Telemedicine field as fast. Attitudes and awareness of telemedicine applications in Sri Lanka were not assessed according to previous literature. The Objective of this study was to determine the level of knowledge, experience and attitude of the general public towards telemedicine in Western Province, Sri Lanka, in order to recommend measures to improve the effectiveness of a telehealth consultation.
\end{abstract}

Methods: A descriptive cross-sectional study done in Western Province, Sri Lanka. The study sample included the general public between the age of 18 years and 64 years, literate in English and IT, and reside in Western Province, Sri Lanka. Participants were selected using random sampling. A self-administered online questionnaire was distributed.

Results: The response rate was $12.1 \%(N=390)$. Majority of the population $(n=257)$ had no previous experience with telemedicine. There was no significant relationship between the use of telemedicine and age $(p=0.54)$, gender $(p=0.703)$, and income $(p=0.786)$. Majority of the people have a good knowledge and a positive attitude towards telemedicine. They find telemedicine is effective and efficient. There were statistically significant relationships between knowledge and perceived improvement in efficiency $(p=0.001)$, knowledge and perceived effectiveness $(p<0.001)$, attitudes and perceived efficiency $(p<0.001) \&$ attitudes and perceived effectiveness $(p<0.001)$.

Conclusions: Age, gender, and income are not barriers to use telemedicine. Better knowledge and positive attitudes improve the perceived effectiveness and efficiency of telemedicine including teleconsultations. Based on the factors that have been tested, spreading and improving the awareness of telemedicine is one of the key measures that can help the growth of telemedicine in Sri Lanka. It is essential for telemedicine service providers to give priority to improve awareness at the same time working on implementations. This study further identifies, taking measures toward greater patient satisfaction is essential for the effective delivery of telemedicine interventions.

Keywords: Telemedicine, Telehealth, Teleconsultations, Telemedicine practices 